\title{
NOTE ON DETERMINANTS OF SINES AND COSINES
}

E. G.-RODEJA F.

In this note we prove the following theorems:

ThEOREM 1. Let

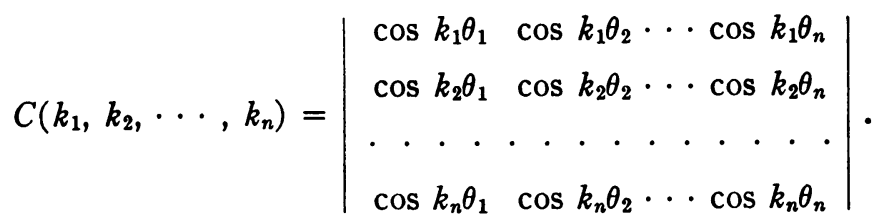

If the $\theta_{j}$ are all real, then for all integers $0 \leqq k_{1}<k_{2}<\cdots<k_{n}$,

$$
\left|\frac{C\left(k_{1}, k_{2}, \cdots, k_{n}\right)}{C(0,1, \cdots, n-1)}\right| \leqq \frac{\prod_{h>j=1}^{n}\left(k_{h}^{2}-k_{j}^{2}\right)}{(n-1) ! 1 ! 3 ! 5 ! \cdots(2 n-3) !}
$$

with equality holding in the limit as all $\theta_{j} \rightarrow 0$.

Theorem 2. Let

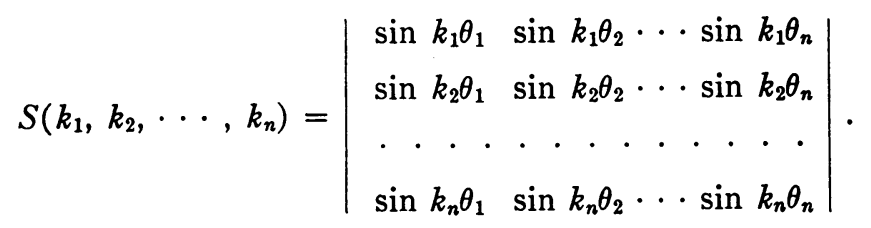

If the $\theta_{j}$ are all real, then for all integers $1 \leqq k_{1}<k_{2}<\cdots<k_{n}$,

$$
\left|\frac{S\left(k_{1}, k_{2}, \cdots, k_{n}\right)}{S(1,2, \cdots, n)}\right| \leqq \frac{\prod_{j=1}^{n} k_{j} \prod_{h>j=1}^{n}\left(k_{h}^{2}-k_{j}^{2}\right)}{1 ! 3 ! 5 ! \cdots(2 n-1) !}
$$

with equality holding in the limit as all $\theta_{j} \rightarrow 0$.

Particular cases of both theorems were proved in a work by A. W. Goodman, ${ }^{1}$ Theorem 1 for $0 \leqq k_{j} \leqq n-1, j=1,2, \cdots, n-1$, and Theorem 2 for $1 \leqq k_{j} \leqq n, j=1,2, \cdots, n-1$.

Received by the editors July 5, 1951.

1 A. W. Goodman, On some determinants related to p-valent functions, Trans. Amer. Math. Soc. vol. 63 (1948) pp. 175-192.

We have already written on a lemma of the same paper in: E. G.-Rodeja F., Note on a lemma of A.W. Goodman, Proceedings of the American Mathematical Society vol. 2 (1951) pp. 314-317. 
Theorems 1 and 2 are similar to the following theorem of Mitchell ${ }^{2}$ on bialternants.

Theorem 3 (Mitchell). Let

$$
V\left(k_{1}, k_{2}, \cdots, k_{n}\right)=\left|\begin{array}{cccc}
z_{1}^{k_{1}} & z_{2}^{k_{1}} \cdots & z_{n}^{k_{1}} \\
z_{2} & z_{2}^{k_{2}} \cdots & z_{n}^{k_{2}} \\
z_{1} & z_{2} & \cdot & \cdot \\
z_{1} & z_{2}^{k_{n}} & \cdots & z_{n}^{k_{n}}
\end{array}\right| .
$$

If

$$
\left|z_{j}\right| \leqq 1, j=1,2, \cdots, n,
$$

then for all integers $0 \leqq k_{1}<k_{2}<\cdots<k_{n}$,

$$
\left|\frac{V\left(k_{1}, k_{2}, \cdots, k_{n}\right)}{V(0,1, \cdots, n-1)}\right| \leqq \frac{\prod_{h>j=1}^{n}\left(k_{h}-k_{j}\right)}{(n-1) !(n-2) ! \cdots 3 ! 2 !}
$$

with equality holding in the limit as all $z_{j} \rightarrow 1$.

For the demonstration of Theorems 1 and 2 we use the following lemmas:

LEMMA 1. Let

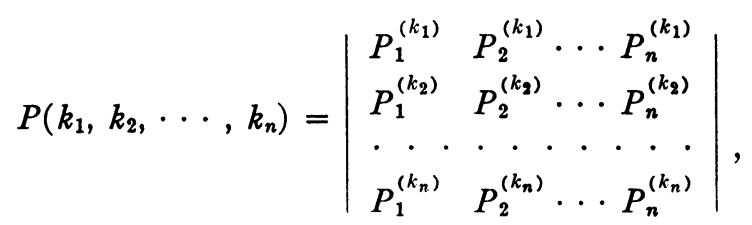

where $P_{j}^{\left(k_{r}\right)}=z_{j}^{\left(k_{r}\right)}+z_{j}^{-k_{r}} . P^{*}(0,1, \cdots, n-1)=(1 / 2) P(0,1, \cdots, n-1) .^{3}$ If $0 \leqq k_{1}<k_{2}<\cdots<k_{n}$, then

$$
\frac{P\left(k_{1}, k_{2}, \cdots, k_{n}\right)}{P^{*}(0,1, \cdots, n-1)}
$$

is a symmetrical polynomial in $P_{1}^{(s)}, P_{2}^{(s)}, \ldots, P_{n}^{(s)}$ with non-negative integer coefficients.

2 O. H. Mitchell, Note on determinants of powers, Amer. J. Math. vol. 4 (1881) pp. 341-344. This theorem and a proof of the same are to be found in Goodmans' work already quoted.

${ }^{3}$ It is useful to consider a determinant $P^{*}(0,1, \cdots, n-1)$ obtained by replacing the first row of $P(0,1, \cdots, n-1)$ formed by twos, $P^{(0)}=2$, by a row of ones. 
LEMma 2. Let

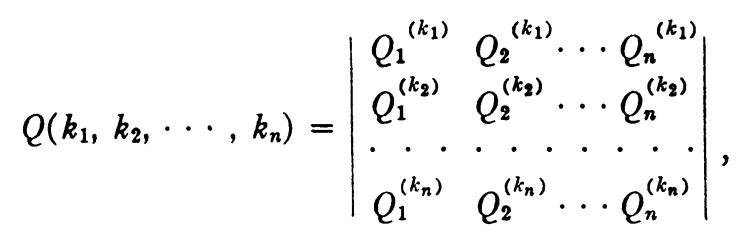

where $Q_{j}^{\left(k_{r}\right)}=z_{j}^{k_{r}}-z_{j}^{-k_{r}}$. If $1 \leqq k_{1}<k_{2}<\cdots<k_{n}$, then

$$
\frac{Q\left(k_{1}, k_{2}, \cdots, k_{n}\right)}{Q(1,2, \cdots, n)}
$$

is a symmetrical polynomial in $P_{1}^{(s)}, P_{2}^{(s)}, \cdots, P_{n}^{(s)}$ with non-negative integer coefficients.

The corresponding lemma for bialternants is well known. ${ }^{4}$ To prove Lemmas 1 and 2, the following lemmas are necessary.

LEMMA 3.5

$$
\begin{aligned}
P^{*}(0,1, \cdots, n-1) & =\prod_{h>j=1}^{n}\left(P_{h}^{(1)}-P_{j}^{(1)}\right), \\
Q(1,2, \cdots, n) & =P^{*}(0,1, \cdots, n-1) \prod_{j=1}^{n} Q_{j}^{(1)}, \\
Q(1,2, \cdots, n) & =\prod_{h>j}^{n}\left(P_{h}^{(1)}-P_{j}^{(1)}\right) \prod_{j=1}^{n} Q_{j}^{(1)} .
\end{aligned}
$$

The first identity of this lemma can be obtained in the same way as that generally used for the development of a Vandermonde determinant, ${ }^{6}$ if we use in this case the identity

$$
\begin{aligned}
P_{h}^{(r)}-P_{j}^{(r)}= & \left(P_{h}^{(1)}-P_{j}^{(1)}\right) P_{h}^{(r-1)}+P_{j}^{(1)}\left(P_{h}^{(r-1)}-P_{j}^{(r-1)}\right) \\
& -\left(P_{h}^{(r-2)}-P_{j}^{(r-2)}\right),
\end{aligned}
$$

easily verifiable if we keep in mind the law of multiplication

$$
P_{j}^{(r)} P_{j}^{(s)}=P_{j}^{(r+s)}+P_{j}^{(r-s)}
$$

4 Mitchell, loc. cit. For a recent proof see P. C. Rosenbloom, Some properties of absolutely monotonic functions, Bull. Amer. Math. Soc. vol. 52 (1946) pp. 458-462.

- The relations of this lemma are equivalent to those obtained by $\mathrm{E}$. Prouhet, Nouvelles Annales de Mathématiques (1) vol. 16 (1857) pp. 403-404 and vol. 17 (1858) pp. 187-190. (See Lemma 3 of the aforesaid paper of Goodman.)

- See, for instance, J. Rey Pastor, Elementos de analisis algebraico, Madrid, 1935, p. 250. 
The second identity is immediately obtained from the identity

$$
Q_{i}^{(s)}=Q_{j}^{(1)} K_{j}(s-1), \quad s=0,1,2, \cdots,
$$

where

$$
K_{j}(s-1)=\sum_{\alpha=0}^{0} P_{j}^{(s-1-2 \alpha)}+2^{-1}\left\{1+(-1)^{8-1}\right\},
$$

and $g=[(s-2) / 2]$

Lemma 4. For $t=0,1,2, \cdots$,

$$
\begin{aligned}
P_{h j}(r, s)= & \left(P_{h}^{(1)}-P_{j}^{(1)}\right) P_{j}^{(s)}\left[\sum_{\alpha=0}^{t} P_{h}^{(r-1-\alpha)} K_{j}(\alpha)\right] \\
& +K_{j}(t+1) P_{h j}(r-t-1, s) \\
& -K_{j}(t) P_{h j}(r-t-2, s),
\end{aligned}
$$

where

$$
P_{h j}(r, s)=\left[P_{h}^{(r)} P_{j}^{(s)}-P_{j}^{(r)} P_{h}^{(s)}\right] .
$$

The proof of this lemma can be attained by induction with respect to $t$, using (15) and the easily proved identity

$$
K_{j}(t+2)=P_{j}^{(1)} K_{j}(t+1)-K_{j}(t) .
$$

Lemma 5. For $t=0,1,2, \cdots$,

$$
\begin{aligned}
Q_{h j}(r, s)= & \left(P_{h}^{(1)}-P_{j}^{(1)}\right) Q_{j}^{(8)}\left[\sum_{\alpha=0}^{t} Q_{h}^{(r-1-\alpha)} K_{j}(\alpha)\right] \\
& +K_{j}(t+1) Q_{h j}(r-t-1, s) \\
& -K_{j}(t) Q_{h j}(r-t-2, s),
\end{aligned}
$$

where

$$
Q_{h j}(r, s)=\left[Q_{h}^{(r)} Q_{j}^{(s)}-Q_{j}^{(r)} Q_{h}^{(s)}\right] .
$$

The proof is similar to that of Lemma 4. For the verification of the identity for $t=0$ it is worthwhile to note the law of multiplication

$$
Q_{j}^{(r)} P_{j}^{(s)}=Q_{j}^{(r+8)}+Q_{j}^{(r-s)} .
$$

For the proof of Lemma 1 we use induction on $n$, the order of the determinant. For $n=1$ the theorem is trivial. The symmetry of the 
polynomial is obvious.

In $P\left(k_{1}, k_{2}, \cdots, k_{n}\right)$ multiply each column except the first by $P_{1}^{\left(k_{1}\right)}$. Then subtract from the $r$ th column $P_{r}^{\left(k_{1}\right)}$ times the first column, for $r=2,3, \cdots, n$. Expansion by minors of the first row gives

$$
\begin{aligned}
& P\left(k_{1}, k_{2}, \cdots, k_{n}\right)
\end{aligned}
$$

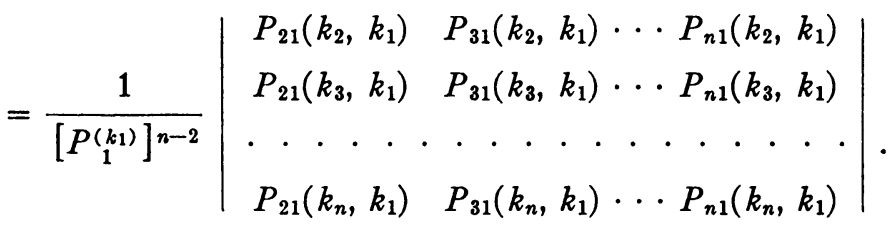

Using the identity of Lemma 4 for $h=2,3, \cdots, n$, with $j=1$, $r=k_{n}, s=k_{1}, t=k_{n}-k_{n-1}-1$, applied to the elements of the last row of (24), we get

$$
\begin{aligned}
& P\left(k_{1}, k_{2}, \cdots, k_{n}\right)=\sum_{\alpha=0}^{k_{n}-k_{n-1}-1} \frac{K_{1}(\alpha)}{\left[P_{1}^{\left(k_{1}\right)}\right]^{n-3}}
\end{aligned}
$$

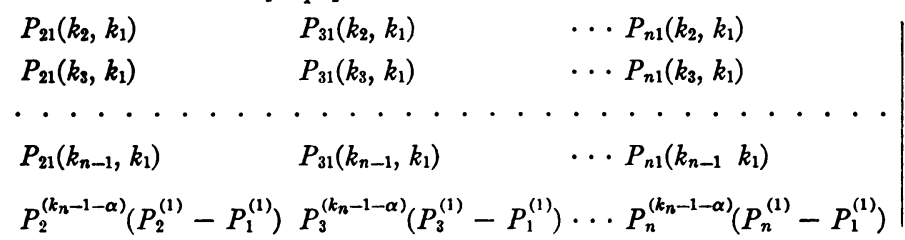

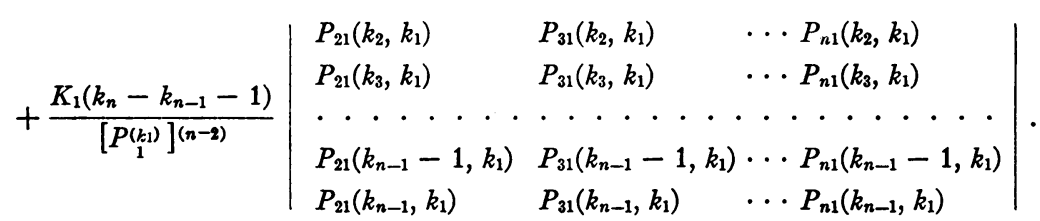

Note that in using (18), the determinant obtained from the second term vanishes, and in the determinant obtained from the last term of (18), the last two rows have been permuted. This determinant also vanishes if $k_{n-2}=k_{n-1}-1$, since two of the rows will then be identical. We point out that in the last row of the first determinant of (25), the terms $P_{h}^{\left(\boldsymbol{k}_{n}-1-\alpha\right)}$ have upper index satisfying the conditions

$$
k_{n-1} \leqq k_{n}-1-\alpha \leqq k_{n}-1 \text {. }
$$

We repeat the same process with each determinant, each time applying the identity of Lemma 4 to the last row formed by elements of the form $P_{h 1}\left(r, k_{1}\right)$ as long as this row is not the first of the determinant.

If we keep in mind that $P_{h 1}\left(k_{1}, k_{1}\right)=0$, we easily observe that the expression obtained for $P\left(k_{1}, k_{2}, \cdots, k_{n}\right)$ is a sum of determinants of 
the form

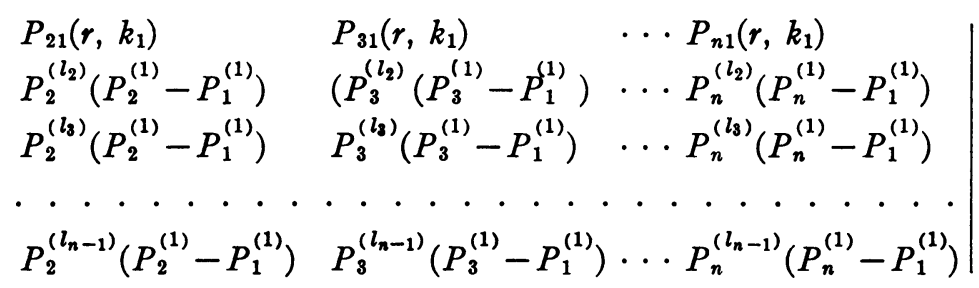

with $k_{1}+1 \leqq r \leqq k_{2}, r \leqq l_{2}<l_{3}<\cdots<l_{n-1} \leqq k_{n}-1$, multiplied by a product of expressions $K_{1}(\alpha)$. The identity

$$
\begin{aligned}
& P_{h j}(r, s)=\left(P_{h}^{(1)}-P_{j}^{(1)}\right)\left[P_{j}^{(s)} \sum_{\alpha=0}^{r-s-2} P_{h}^{(r-1-\alpha)} K_{j}(\alpha)\right. \\
& \left.\quad+K_{j}(r-s-1)\left(\sum_{\alpha=0}^{s-1} P_{h}^{(s-\alpha)} P_{j}^{(s-\alpha)}+2\right)\right], r \geqq s+2,
\end{aligned}
$$

is easily obtained from Lemma 4 , by setting $t=r-s-2$, and noting that

$$
P_{h j}(s+1, s)=\left(P_{h}^{(1)}-P_{j}^{(1)}\right)\left[\sum_{\alpha=0}^{s-1} P_{h}^{(8-\alpha)} P_{j}^{(8-\alpha)}+2\right] .
$$

A direct computation shows that (27) is also valid when $r=s+1$.

Using (27) in the first row of (26), taking off a common factor of each column $\left(P_{h}^{(1)}-P_{1}^{(1)}\right), h=2,3, \cdots, n$, dividing these determinants and $P^{*}(0,1, \cdots, n-1)$ by $\prod_{h=2}^{n}\left(P_{h}^{(1)}-P_{1}^{(1)}\right)$, keeping in mind the first relation of Lemma 3 , we obtain an expression for $P\left(k_{1}, k_{2}, \cdots, k_{n}\right): P^{*}(0,1,2, \cdots, n-1)$ as an addition of products of polynomials of $P_{1}^{(s)}$ by similar quotients of determinants of order $n-1$. The proof of Lemma 1 by induction is thus finished.

The proof of Lemma 2 is similar to the preceding one, using Lemma

5. The identities replacing (27) and (28) are

$$
\begin{aligned}
Q_{h j}(r, s) & =\left(P_{h}^{(1)}-P_{j}^{(1)}\right)\left[Q_{j}^{(s)} \sum_{\alpha=0}^{r-s-2} Q_{h}^{(r-1-\alpha)} K_{j}(\alpha)\right. \\
+ & \left.K_{j}(r-s-1)\left(\sum_{\alpha=0}^{s-1} Q_{h}^{(8-\alpha)} Q_{j}^{(8-\alpha)}\right)\right], \quad r \geqq s+1, \\
Q_{h j}(s+1, s) & =\left(P_{h}^{(1)}-P_{j}^{(1)}\right)\left[\sum_{\alpha=0}^{s-1} Q_{h}^{(8-\alpha)} Q_{j}^{(8-\alpha)}\right] .
\end{aligned}
$$

The only difference in proving Lemma 2 is that the expression (29) ought to be written, by using the identity (16), thus: 


$$
\begin{aligned}
Q_{h j}(r, s)=\left(P_{h}^{(1)}\right. & \left.-P_{j}^{(1)}\right) Q_{j}^{(1)}\left[K_{j}(s-1) \sum_{\alpha=0}^{r-s-2} Q_{h}^{(r-1-\alpha)} K_{j}(\alpha)\right. \\
& \left.+K_{j}(r-s-1)\left(\sum_{\alpha=0}^{s-1} Q_{h}^{(s-\alpha)} K_{j}(s-\alpha-1)\right)\right],
\end{aligned}
$$

and using this expression for the elements of the first row of determinants (in which $j=1$ ), we can get a common factor $Q_{1}^{(1)}$, by which these determinants and $Q(1,2, \cdots, n)$ will be divided, thus obtaining, keeping in mind the third relation of Lemma 3, an expression for

$$
Q\left(k_{1}, k_{2}, \cdots, k_{n}\right): Q(1,2, \cdots, n)
$$

as a sum of products of polynomials of $P_{1}^{(s)}$ by similar quotients of determinants of order $n-1$. Lemma 2 is also established.

From Lemmas 1 and 2 we deduce that for values of $z_{j}, j=1,2, \cdots$, $n$, belonging to the ring domain $\delta_{1} \leqq\left|z_{j}\right| \leqq \delta_{2}$, the quotients $P\left(k_{1}, k_{2}, \cdots, k_{n}\right): P^{*}(0,1, \cdots, n-1)$ and $Q\left(k_{1}, k_{2}, \cdots, k_{n}\right)$ : $Q(1,2, \cdots, n)$ assume maximum absolute value when all the $z_{j}$ are equal either to $\delta_{1}$ or to $\delta_{2}$ according as $\delta_{1}+\delta_{1}^{-1} \gtrless \delta_{2}+\delta_{2}^{-1}$.

For $\left|z_{j}\right|=1, j=1,2, \cdots, n$, the maximum absolute value of the said quotients is the value for $z_{j}=1, j=1,2, \cdots, n$. For these values of the $z_{j}$ the numerator and denominator both vanish. It will therefore be necessary to apply the rule of L'Hospital to calculate this maximum.

For the calculation of the value of the quotient $P\left(k_{1}, k_{2}, \cdots, k_{n}\right)$ : $P^{*}(0,1, \cdots, n-1)$ it is necessary to differentiate the numerator and denominator $2(j-1)$ times with respect to each variable $z_{j}$ (the derivatives of odd order of $P_{j}^{(k)}$ vanish for $\left.z_{j}=1\right)$.

The 2(j-1)-derivative of $P_{j}^{(k)}$ for $z_{j}=1$,

$$
\prod_{\alpha=0}^{2 j-3}(k-\alpha)+\prod_{\alpha=0}^{2 j-3}(k+\alpha),
$$

is an even polynomial in $k$ of degree $2 j-2$, with coefficient 2 for the term of highest degree. After the process of differentiating, the numerator can be transformed into the product of $2^{n}$ by a Vandermonde determinant in the numbers $k_{1}^{2}, k_{2}^{2}, \cdots, k_{n}^{2}$, and in the same way the denominator is the product of $2^{n-1}$ by a Vandermonde determinant in the numbers $0,1^{2}, 2^{2}, \cdots,(n-1)^{2}{ }^{2}$

Therefore, for $\left|z_{j}\right|=1, j=1,2, \cdots, n$,

7 The difference between the exponents of 2 in the numerator and denominator is a consequence of the definition of $P^{*}(0,1, \cdots, n-1)$. 


$$
\left|\frac{P\left(k_{1}, k_{2}, \cdots, k_{n}\right)}{P^{*}(0,1, \cdots, n-1)}\right| \leqq \frac{2 \prod_{h>j=1}^{n}\left(k_{h}^{2}-k_{j}^{2}\right)}{(n-1) ! 1 ! 3 ! 5 ! \cdots(2 n-3) !} .
$$

For the calculation of the value of the quotient $Q\left(k_{1}, k_{2}, \cdots, k_{n}\right)$ : $Q(1,2, \cdots, n)$ it is necessary to differentiate the numerator and the denominator $2 j-1$ times with respect to each variable $z_{j}$. The $(2 j-1)$ derivative of $Q_{j}^{(k)}$ for $z_{j}=1$,

$$
\prod_{\alpha=0}^{2 j-2}(k-\alpha)+\prod_{\alpha=0}^{2 j-2}(k+\alpha)
$$

is an odd polynomial in $k$ of degree $2 j-1$ with coefficient 2 for the term of highest degree. After the process of differentiating, the numerator can be transformed into the product of $2^{n} \prod_{j=1}^{n} k_{j}$ by a Vandermonde determinant in the numbers $k_{1}^{2}, k_{2}^{2}, \cdots, k_{n}^{2}$ and in the same way the denominator is the product of $2^{n} \cdot n$ ! by a Vandermonde determinant in the numbers $1^{2}, 2^{2}, \cdots, n^{2}$.

Therefore for $\left|z_{j}\right|=1, j=1,2, \cdots, n$,

$$
\left|\frac{Q\left(k_{1}, k_{2}, \cdots, k_{n}\right)}{Q(1,2, \cdots, n)}\right| \leqq \frac{\prod_{j=1}^{n} k_{j} \prod_{h>j=1}^{n}\left(k_{h}^{2}-k_{j}^{2}\right)}{1 ! 3 ! 5 ! \cdots(2 n-1) !} .
$$

From $z_{j}=\cos \theta_{j}+i \sin \theta_{j},\left|z_{j}\right|=1, \cos \theta_{j}=(1 / 2) P_{j}^{(1)} \sin \theta_{j}=(1 / 2 i) Q_{j}^{(1)}$, $z_{j}=1$ for $\theta_{j}=0$, we get Theorems 1 and 2 as an immediate consequence of the inequalities (33) and (35).

Santiago de Compostela 